\title{
Auditory fear conditioning increases CS-elicited spike firing in lateral amygdala neurons even after extensive overtraining
}

\author{
Stephen Maren \\ Department of Psychology and Neuroscience Program, University of Michigan, 525 E. University Ave., Ann Arbor, \\ MI 48109-1109, USA
}

Keywords: basolateral amygdala, electrophysiology, freezing, neuronal plasticity, parallel recording, Pavlovian conditioning

\begin{abstract}
We examined the influence of extensive overtraining (75 trials) of auditory fear conditioning on the expression of conditional stimulus (CS)-elicited spike firing in lateral amygdala (LA) neurons. Single units were recorded from chronic multichannel electrodes implanted in the LA of conscious and freely moving rats. In sequential training sessions, the rats received either five or 70 fear conditioning trials, which consisted of a white-noise CS and a coterminating footshock unconditional stimulus (US). Unpaired (sensitization) controls received the same number of trials, but the CS and US were explicitly unpaired. Paired CS-US presentations yielded robust increases in CS-elicited spike firing in LA neurons after both five and 70 conditioning trials, and the magnitude of the spike firing increases was correlated with the expression of conditional freezing to the CS. After 75 training trials, maximal conditioning-related increases in LA firing were exhibited within $20 \mathrm{~ms}$ of CS onset, indicating that this increase is mediated by direct thalamo-amygdala projections. There was no significant increase in CS-elicited spike firing or freezing behaviour in the unpaired group. These results complement amygdala lesion studies [e.g. Maren, S. (1999a) J. Neurosci., 19, 8696-8703] and support the view that the basolateral complex of the amygdala is involved in the encoding and storage of fear memories even after extensive overtraining.
\end{abstract}

\section{Introduction}

In Pavlovian fear conditioning, animals learn that an innocuous stimulus (the conditional stimulus or CS), such as a tone, predicts the occurrence of an aversive stimulus (the unconditional stimulus or US), such as an electric footshock. Conditional fear is manifested by a variety of behavioural responses, including freezing (somatomotor immobility). Several laboratories have now demonstrated that both the acquisition and expression of conditional fear require neurons in the basolateral complex of the amygdala (BLA) (see LeDoux, 2000 for a review). Indeed, the BLA appears to be necessary for the expression of conditional freezing up to one month following fear conditioning (Lee etal., 1996; Maren etal., 1996a) and after extensive overtraining (Maren, 1998; 1999a). These findings have led many investigators to propose that the BLA has a role in the encoding and long-term storage of fear memories (Davis, 1997; Maren \& Fanselow, 1996; Fanselow \& LeDoux, 1999; Maren, 1999b; LeDoux, 2000).

Consistent with a role for the BLA in encoding fear memories, it has been reported that aversive conditioning increases auditory CSelicited spike firing in neurons in the BLA (Maren et al., 1991; Quirk et al., 1995, 1997). However, learning-related changes in amygdaloid neuronal activity have been reported to dissipate with extended training under some conditions. For example, we have reported that associative neuronal activity in the BLA shows a persistent decrease after extensive overtraining in an avoidance-conditioning task in

Correspondence: Stephen Maren, as above.

E-mail: maren@umich.edu

Received 17 April 2000, revised 10 August 2000, accepted 14 August 2000 rabbits (Maren et al., 1991). It has also been reported that associative activity in the lateral nucleus of the amygdala (LA) decreases over the course of several conditioning trials in a Pavlovian fear conditioning task (Quirk et al., 1997). In this case, however, associative increases in CS-elicited spike firing are reinstated during shock-free extinction testing one hour after conditioning. A similar phenomenon has been observed in neuroimaging studies of amygdaloid blood flow during aversive conditioning (Buchel et al., 1998; LaBar etal., 1998). This pattern of results suggests that the BLA may have only a temporary role in mediating aversive conditioning, perhaps modulating memory storage elsewhere in the brain during the early stages of training (e.g. Cahill \& McGaugh, 1998; McGaugh, 2000). Consistent with this view, there is evidence to support temporary involvement of the amygdala in instrumental avoidance conditioning (Liang et al., 1982; Parent et al., 1992, 1994; Poremba \& Gabriel, 1999).

The pattern of amygdaloid spike firing during the course of aversive conditioning has important implications for understanding the role of amygdaloid neurons in this form of learning. Insofar as associative neuronal firing in the amygdala indexes memory encoding and/or storage, one would expect learning-related spike firing in the BLA to be manifested under conditions in which the BLA is known to be important for conditioning. Because we have recently demonstrated that the BLA is essential for Pavlovian fear conditioning to auditory CSs after extensive overtraining (Maren, 1998; Maren, 1999a), it is critical to systematically assess whether learning-related increases in CS-elicited spike firing are evident in the amygdala after extensive overtraining. To this end, we have used multichannel single-unit recording techniques to examine the influence of extensive overtraining (75 trials) on the expression of conditional 
plasticity in LA neurons in an auditory fear-conditioning paradigm. This level of training is more than sufficient to produce asymptotic levels of conditional freezing (Maren, 1999a). We recorded from neurons in the dorsal division of the lateral nucleus (LAd), a region of the LA that contains a high density of auditory-responsive neurons (Quirk et al., 1995).

\section{Materials and methods}

\section{Subjects, surgery and electrodes}

The subjects were nine adult male Long-Evans rats (200-450 g) obtained from a commercial supplier (Harlan Sprague-Dawley, Indianapolis, IN, USA). The rats were individually housed in standard stainless-steel hanging cages on a 14:10-hr light: dark cycle (lights on at $07.00 \mathrm{~h}$ ), provided with free access to food and tap water, and handled daily. All of the experimental procedures involving the subjects were approved by the University Committee on Use and Care of Animals (UCUCA) at the University of Michigan.

Our recording probes and procedures for single-unit recording in the amygdala were adapted from Quirk et al. (1995). Under sodium pentobarbital anaesthesia, a multichannel recording probe was implanted in the LA $(3.3 \mathrm{~mm}$ posterior and $5.5 \mathrm{~mm}$ lateral to bregma, $6.0-6.5 \mathrm{~mm}$ ventral to dura). The probe consisted of a twisted bundle of eight tungsten wires $(25 \mu \mathrm{M}$ diameter, $100-200 \mathrm{k} \Omega$ impedance at $1 \mathrm{kHz}$; California Fine Wire, Grover Beach, CA, USA) extending $1 \mathrm{~mm}$ beyond the tip of a $28 \mathrm{~g}$ guide cannula. One wire served as a reference from which differential recordings were made on the other active wires. The wires were soldered to a 20-pin $(10 \times 2)$ Mill-Max connector (Mill-Max Manufacturing, Oyster Bay, NY, USA) and the wire and cannula assembly was affixed to the connector with dental acrylic. The probe was aimed at the dorsal division of the LAd and was positioned by monitoring auditory-evoked unit discharges. Four jeweler's screws were attached to the skull. One screw served as the animal ground and the others were used to anchor the electrode assembly. The entire electrode assembly was affixed to the skull with dental acrylic. The low-impedance electrodes we used are not optimal for single-unit isolation due to the relatively low signal-to-noise ratio they yield. However, they did permit us to record from single neurons over a 3-day period, which was demanded by our behavioural paradigm (see below).

\section{Apparatus and procedure}

A modified rodent conditioning chamber $(30 \times 24 \times 21 \mathrm{~cm}$; MEDAssociates Inc., Burlington, VT, USA) was used for all behavioural and neurophysiological testing. The chamber was constructed from aluminium (side walls) and Plexiglas (rear wall and door). The top of the chamber was removed and all of the chamber walls were extended $19 \mathrm{~cm}$ with Plexiglas to prevent the rats from exiting via the open top of the chamber; the open top accommodated the recording cable and commutator. The chamber was situated in a sound-attenuating cabinet, which was located in a quiet, dark room. A $1 \%$ acetic acid solution was used to clean the chamber between subjects, and a thin film of this solution was placed in the pan underneath the grid floor [19 stainless steel rods (4-mm diameter) spaced $1.5 \mathrm{~cm}$ apart (centre-to-centre)]. The chamber was equipped with a speaker for the delivery of auditory CSs (white noise, $85 \mathrm{~dB}, 2 \mathrm{~s}$ ) and a grid floor for the delivery of scrambled footshock USs $(1.0 \mathrm{~mA}, 0.5 \mathrm{~s})$. Freezing behaviour was continuously monitored via an automated system (see Maren, 1999a).

After a 3-day recovery from surgery, the rats were transported to the conditioning chamber for the first of three 10-min recording sessions, which were conducted over a 3-day period (one session per day). Before each recording session, a headstage cable consisting of two four-channel operational amplifiers (TLC2274ACD; Texas Instruments, Dallas, TX, USA) in a unity-gain (source-follower) configuration was connected to the electrode assembly on each rat's head. The operational amplifiers were battery-powered ( $\pm 6 \mathrm{~V} \mathrm{DC})$. The cable was connected to a commutator, which permitted the rats to move freely within the conditioning chamber. During each recording session the rats were presented with 10 white-noise stimuli $(85 \mathrm{~dB}$, $2 \mathrm{~s}, 1$-min ISI). Neuronal data were amplified $(10000 \times)$ and filtered (0.6-6 kHz) (Neuralynx, Tuscon, AZ, USA) and acquired and digitized $(32 \mathrm{kHz} /$ channel) with Experimenter's Workbench software (DataWave Technologies, Longmont, CO, USA) during a 3-s period ( $0.5 \mathrm{~s}$ before, $2 \mathrm{~s}$ during and $0.5 \mathrm{~s}$ after each white-noise stimulus). Freezing data were continuously acquired during both the white-noise stimuli and the ISIs.

The neuronal recording and fear conditioning sessions for each rat were carried out in the following manner: Day 1, recording session followed $6 \mathrm{~h}$ later by five conditioning trials; Day 2, recording session followed $6 \mathrm{~h}$ later by 70 conditioning trials; Day 3 , recording session. On Days 1 and 2, the rats were returned to their home cages in between the recording and conditioning sessions. Thus, the three recording sessions yielded pretraining data on Day 1 and postconditioning data after fve and 75 trials on Days 2 and 3, respectively. For rats in the 'paired' group $(n=5)$, the footshock US coterminated with the white-noise CS during the fear conditioning sessions, whereas the white-noise CS and footshock US were explicitly unpaired for rats in the 'unpaired' group $(n=4)$. Neuronal data were not recorded during the conditioning sessions due to electrical noise from the footshock and shock-elicited locomotor activity. This design allowed a within-subjects comparison of LAd spike firing after five and 75 trials in individual rats, and allowed a between-subjects comparison of the influence of CS/US contingency on LAd spike firing.

\section{Data analysis}

Neuronal data were analysed off-line using Experimenter's Workbench and Autocut software (DataWave). Single units were isolated on each recording channel using window discriminators and spike clustering algorithms. Auto- and cross-correlograms and interspike interval histograms were used to verify that isolated single-unit waveforms on each recording channel were generated by individual LAd neurons. Cluster boundaries were computed from the second recording session and applied to the first and third recording sessions. We did not attempt to adjust the cluster boundaries from session to session; therefore some spikes were lost due to movement of the brain and electrodes. However, in many cases, single units could be tracked over the 3-day recording period. Units that were not stable over this recording period were excluded. All unit data were binned (50-ms) and normalized to the 500-ms pre-CS baseline. For each recording session, peri-stimulus time histograms (PSTHs) were summed over the 10 trials and an average PSTH was computed for all units in each group of rats. The statistical analysis was limited to auditory-responsive neurons, defined by having a response $>3 \mathrm{SD}$ above the pre-CS mean in the first post-CS bin during any of the three recording sessions.

The freezing data for each session were transformed to a percentage of total observations. Neuronal and behavioural data were analysed using analysis of variance (ANOvA). Post hoc comparisons in the form of Fisher Least Significant Difference tests were performed following a significant omnibus $F$-ratio. All data are represented as means \pm SEM. 


\section{Histology}

After behavioural testing, a marking lesion was made at the tip of one electrode by passing anodal current $(80 \mu \mathrm{A}, 10 \mathrm{~s})$. The rats were perfused across the heart ( $0.9 \%$ saline followed by $10 \%$ formalin) and the brain was removed and postfixed in $10 \%$ formalin for 2 days and $30 \%$ sucrose $10 \%$ formalin until sectioning. The brain was frozen and sectioned $(50 \mu \mathrm{M})$ on a cryostat $\left(-17^{\circ} \mathrm{C}\right)$. The sections were stained with $0.25 \%$ thionin to visualize neuronal cell bodies and the electrode tracks. Electrode placements were verified by reconstructing the electrode tracks on stereotaxic atlas templates.

\section{Results}

\section{Histology}

As shown in Fig. 1A and B, the recording probes were located primarily in LAd, although one probe was located in the ventral division of LA and two probes were placed in the amygdalo-striatal transition zone (AST) immediately adjacent to LAd. The distribution of placements did not differ across the groups. Auditory responsive neurons were present in all of these regions, and the firing properties of cells in these regions did not differ markedly (see also Quirk et al., 1995). We therefore included neurons recorded from LAd, LAv and AST in the analyses described below.

\section{Electrophysiology}

A total of 270 cells (average pre-CS and pretraining firing rate, $8.0 \mathrm{~Hz}$; range, $0.2-50.8 \mathrm{~Hz}$ ) were recorded from rats in the paired (141 cells) and unpaired (129 cells) groups across the three recording sessions; $56.3 \%$ of these cells were responsive to the white-noise CS at a short latency $(50 \mathrm{~ms})$ during at least one of the three recording sessions (76 cells in each group). In the paired group, white-noiseresponsive cells were recorded from the LAd $(n=60)$, LAv $(n=5)$ and AST $(n=11)$. In the unpaired group, white-noise-responsive cells were recorded from the LAd $(n=56)$ and AST $(n=20)$. The statistical analysis reported below focuses on this population of auditoryresponsive cells.

Figure 1C illustrates the PSTHs for each recording session and corresponding averaged spike waveforms of a single LAd neuron recorded in a rat from the paired group (electrode placement shown in Fig. 1A). The response pattern for this cell was representative of others in its group. The cell exhibited a long-latency auditory response $(>50 \mathrm{~ms})$ to the white-noise CS prior to fear conditioning, and acquired a short-latency $(<50 \mathrm{~ms})$ response to the CS after five conditioning trials. This conditioning-related increase in the CSelicited response persisted even after 70 additional training trials. Note that the spike waveform of the neuron remained relatively stable across the three recording sessions.

The mean $z$-scores for all of the cells recorded in the paired and unpaired groups are shown in Fig. 2. An ANOVA with factors of group and session performed on the short-latency bin $(0-50 \mathrm{~ms})$ revealed that CS-elicited firing in the paired and unpaired groups varied differently across the three recording sessions (group-session interaction: $\left.F_{2,300}=4.0, P<0.02\right)$. Post hoc comparisons $(P<0.05)$ indicated that short-latency conditional activity in the paired group increased after five conditioning trials and remained elevated after 70 additional trials. In contrast, there was no significant change in CSelicited firing among cells from unpaired rats. Interestingly, cells from both paired and unpaired rats showed a shift in maximal firing
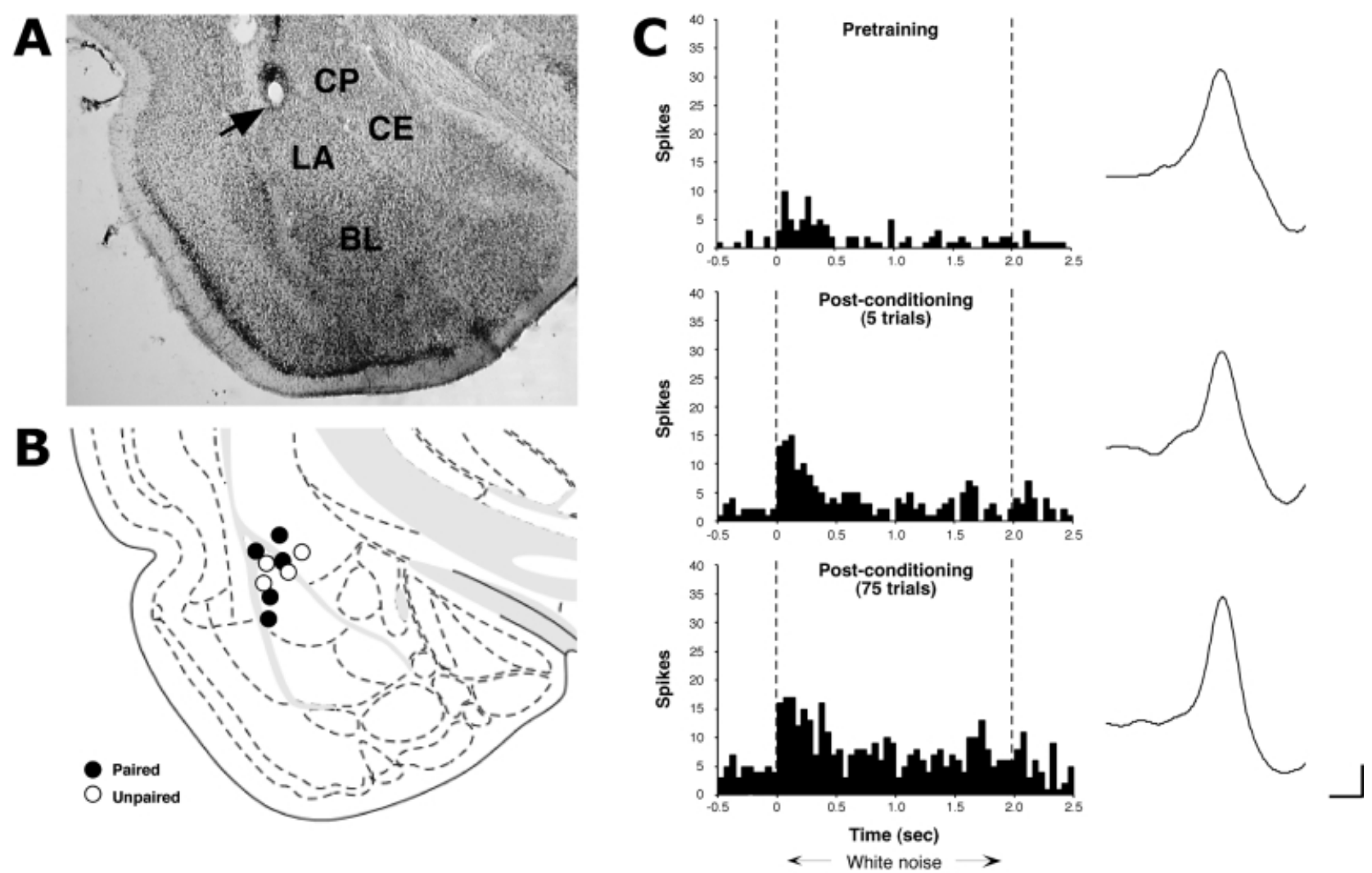

FIG. 1. (A) Photomicrograph of a thionin-stained brain section from a representative rat in the paired group. (B) Schematic representation of electrode placements in paired (filled circles) and unpaired (open circles) rats. (C) Peri-stimulus time histograms (PSTHs, spikes summed over 10 trials per session) and average spike waveforms for the three recording sessions from the rat whose electrode placement is illustrated in A. Fear conditioning increased spike firing in the short-latency $(0-50 \mathrm{~ms})$ bin; the spike waveform was stable across the 3 days of recording. 

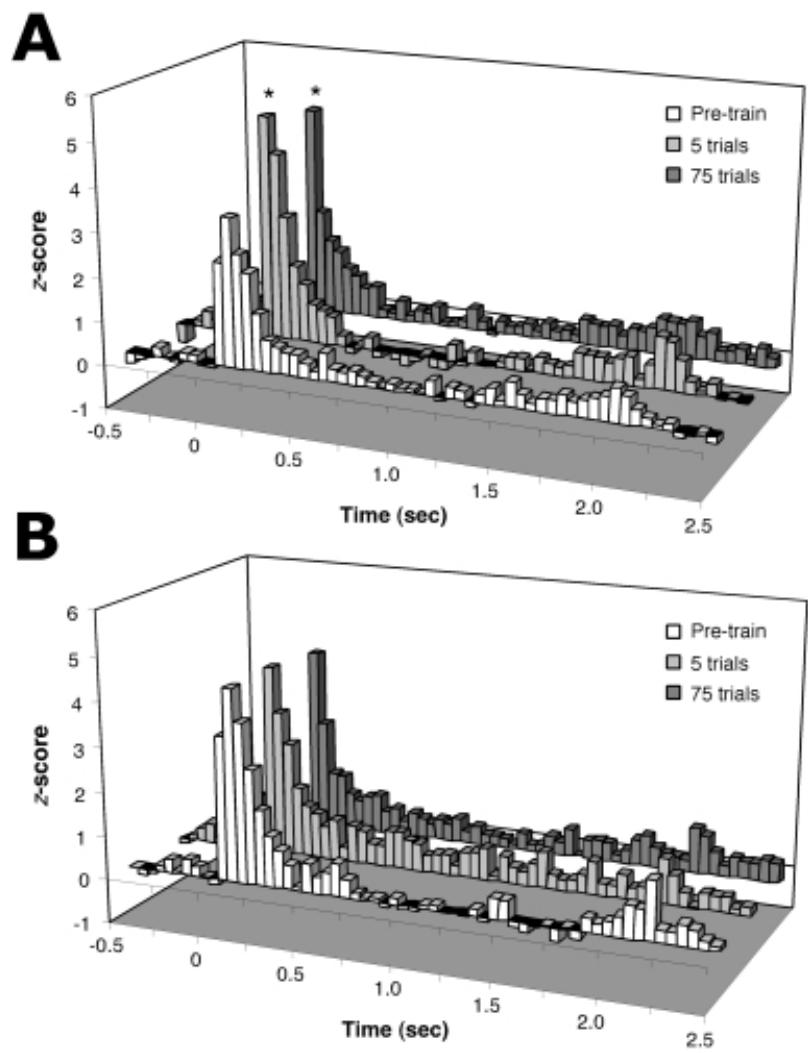

FIG. 2. Mean peri-stimulus time histograms (PSTHs, spikes summed over 10 trials per session and normalized to the pre-CS baseline; 50-ms bins) for the three recording sessions (pretrain, five trials, 75 trials) for all auditoryresponsive cells recorded from rats in the paired (A) and unpaired (B) groups ( $n=76$ cells/group). The $z$-scores (mean \pm SEM) in the first 50 -ms bin for each group and session were as follows: paired: Pre, $2.36 \pm 0.28$; five trials, $5.10 \pm 0.51 ; 75$ trials, $4.78 \pm 0.33$; unpaired: Pre, $3.25 \pm 0.34$; five trials, $4.26 \pm 0.41 ; 75$ trials, $4.10 \pm 0.33$.

from long-latency $(50-100 \mathrm{~ms})$ to short-latency $(0-50 \mathrm{~ms})$ bins; however, only cells from the paired rats actually increased their firing rate in the short-latency bin. Overall firing rate in the shortlatency bin was not significantly greater in the paired rats than in the unpaired rats (group: $F_{1,150}=0.33$ ); however, this was largely due to the different levels of pretraining CS-evoked activity in the two groups. That is, CS-elicited firing in the paired groups was found to be statistically greater than that in the unpaired rats when the CSelicited neuronal firing during the postconditioning test sessions was normalized to the pretraining session.

It is unlikely that the greater pretraining CS-elicited activity in the unpaired rats accounted for their failure to exhibit conditioningrelated increases in CS-elicited firing. Indeed, we observed robust conditioning-related increases in CS-elicited firing in paired rats with levels of pretraining CS-elicited activity greater than that in the unpaired rats described above. More specifically, we recorded CSelicited spike firing from LAd in two additional rats (a total of 34 cells) both before and after a single training session consisting of 75 noise-footshock trials. The average firing rate (mean $z$-score \pm SEM) in the first bin after CS-onset $(0-50 \mathrm{~ms}$ bin) was $5.09 \pm 0.66$ before conditioning and $8.02 \pm 1.03$ after conditioning; the magnitude of these $z$-scores was statistically different $\left(t_{33}=3.45, P<0.01\right)$. This reveals that robust pretraining CS-elicited spike firing in LAd neurons does not impede the development of conditioning-related increases in CS-elicited spike firing.
The average firing rate we recorded in our population of LAd neurons was relatively high compared to other published reports of LAd activity (e.g. Quirk et al., 1995). This suggests that we may have sampled from a greater number of fast-firing inhibitory interneurons than in previous reports. Alternatively, we may have sampled from multiple units, rather than single neurons, in some cases. To examine whether firing rate affected the outcome of our results, we performed a median split on the unit data based on the firing rate of cells in each group of rats and included rate as a variable in the ANOVA described above. This allowed us to analyse CS-elicited spike firing in both slow firing $(2.8 \pm 0.2 \mathrm{~Hz})$ and fast firing $(16.9 \pm 1.3 \mathrm{~Hz})$ LAd neurons, which are presumably pyramidal neurons and inhibitory interneurons, respectively. In no case did the firing-rate variable yield significant effects in the ANOVA.

Our method of single-unit recording in the present study is based on recording methods used in recently published studies of amygdaloid unit activity (e.g. Quirk etal., 1995, 1997). It is important to note, however, that these recording methods do not permit the same degree of unit isolation that can be obtained with stereotrode or tetrode techniques (e.g. McNaughton et al., 1983; Wilson \& McNaughton, 1993). In view of these limitations, we have made strong efforts to exclude redundant recordings of individual units on multiple recording wires. However, it remains possible that we overestimated the number of unique neurons from which we have recorded in the present study. We examined whether this might impact on our conclusions by limiting our statistical analyses of the short-latency $(0-50 \mathrm{~ms})$ post-CS onset bin to the units obtained from only one recording channel per rat (for each rat we selected the channel with the most units). Under these conditions, the number of units included in the analysis dropped to $25 \%$ of our original sample ( $n=19$ cells per group). However, the pattern of unit firing remained the same: short-latency firing increased after either five or 70 conditioning trials in the paired group, and there were no changes in firing in the unpaired group (group-session interaction: $F_{2,72}=4.47$, $P<0.02$ ). Therefore, it is not likely that redundant sampling of LA neurons (if it was present in our data set) contributed substantially to the pattern of results that we observed.

Conditioning-related changes in LAd neuronal firing could arise from plasticity in afferent projections from either the medial geniculate nucleus of the thalamus or the auditory cortex (by way of the medial geniculate nucleus) to the LAd. It has been argued that plasticity in these two pathways can be discriminated, in part, based upon the latency from CS onset at which plasticity in LAd spike firing is first evident (e.g. Quirk et al., 1997). Specifically, conditioninginduced increases in LAd spike firing within $20 \mathrm{~ms}$ of CS onset would be consistent with plasticity in the thalamo-amygdala pathway, whereas plasticity between 20 and $50 \mathrm{~ms}$ after CS onset would be consistent with plasticity in either the thalamo-amygdala or thalamocortico-amygdala pathway. Therefore, we re-analysed our data using shorter $10-\mathrm{ms}$ bins to increase temporal resolution within the first $100 \mathrm{~ms}$ of the white-noise CS.

As shown in Fig. 3A and B, conditioning-related increases in the paired group were first evident within $20 \mathrm{~ms}$ of CS onset, consistent with plasticity in thalamic projections to LAd. Interestingly, the pattern of CS-elicited increases in LAd firing varied as a function of training. An ANOVA with factors of training session and post-CS bin revealed a significant interaction between these variables $\left(F_{8,600}=6.38, P<0.0001\right)$. Post hoc comparisons $(P<0.05)$ revealed that after five conditioning trials, plasticity was evident in both shortand long-latency bins $(10-60 \mathrm{~ms})$. However, after 75 conditioning trials, significant conditioning-related increases in CS-elicited spike firing were limited to only short-latency bins $(10-30 \mathrm{~ms})$. This pattern 

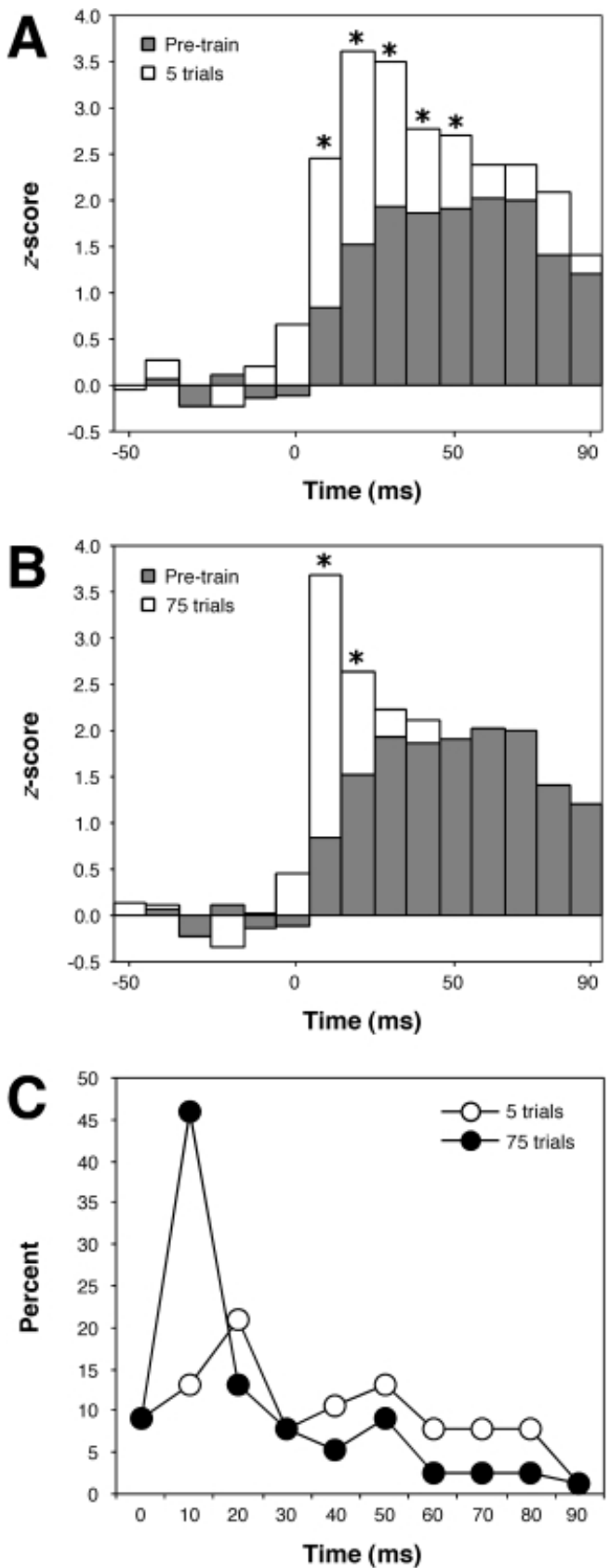

FIG. 3. (A) Mean peri-stimulus time histograms (PSTHs, spikes summed over 10 trials per session and normalized to the pre-CS baseline; $10-\mathrm{ms}$ bins) for two recording sessions (pretrain and five trials) for all auditory-responsive cells recorded from rats in the paired group ( $n=76$ cells). (B) Mean PSTHs (spikes summed over 10 trials per session and normalized to the pre-CS baseline; $10-\mathrm{ms}$ bins) for two recording sessions (pretrain and 75 trials) for all auditory-responsive cells recorded from rats in the paired group ( $n=76$ cells). (C) Percentage of cells recorded from rats in the paired group that exhibited maximal conditioning-related increases in spike firing in each postshock session. For this measure, $z$-scores from the preshock session were subtracted from $z$-scores from each postshock session, and the post-CS onset bin with the largest difference score was used to define the latency at which peak conditioning-related firing occurred. Subtractions were performed on a bin-bybin basis for ten post-CS onset bins. Note the strong shift in peak conditioningrelated firing to the $10-20 \mathrm{~ms}$ bin after extensive overtraining.

of results was also evident in the large percentage of cells that exhibited their peak conditioning-related increase in CS-elicited firing in the $10-20 \mathrm{~ms}$ bin after extensive overtraining (Fig. 3C). Thus, extensive overtraining sharpens conditioning-related increases

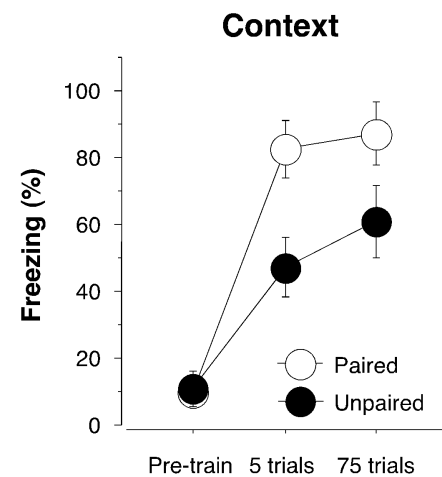

\section{White Noise}

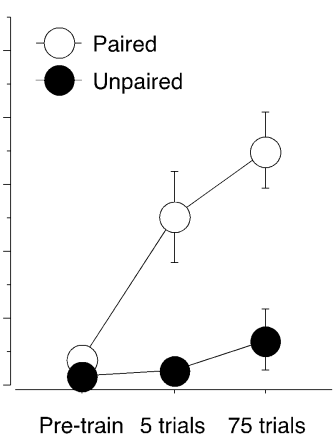

FIG. 4. Mean ( \pm SEM) percentage of freezing during the interstimulus intervals (context) and white-noise CSs (white noise).

in LAd firing within the short-latency bins. This suggests that plasticity develops in both thalamic (short-latency) and auditory cortical (long-latency) projections to the LAd early in auditory fear conditioning (within five trials), but is restricted to thalamo-amygdala projections after more extensive training. There were no significant changes in CS-elicited firing within $100 \mathrm{~ms}$ of CS onset in rats receiving unpaired training (data not shown).

\section{Behaviour}

The increase in CS-elicited spike firing in the paired rats was correlated with the acquisition of conditional freezing (Fig. 4). Both paired and unpaired rats exhibited freezing to the contextual cues associated with footshock, and paired rats exhibited greater levels of contextual freezing than unpaired rats (group: $F_{1,7}=6.2, P<0.05$ ). Importantly, only paired rats acquired conditional freezing to the white-noise CS. This was indicated by a significant interaction of group and training in the ANOvA $\left(F_{2,14}=7.1, P<0.01\right)$; post hoc comparisons $(P<0.05)$ revealed that rats in the unpaired group did not exhibit a significant increase in conditional freezing after either five or 75 conditioning trials. Thus, despite showing conditional freezing to the context of the conditioning chamber, rats in the unpaired group did not acquire conditional freezing to the white-noise CS (Fig. 4).

In paired rats, freezing to the conditioning context was slightly higher than freezing to the CS even after 75 noise-shock trials. This is probably due to the fact that freezing to the white-noise CS was sampled during the short (2-s) duration of the CS (compared to the longer 60-s periods used to sample context freezing), and it is difficult to quantify freezing behaviour over such short sampling intervals. During this interval, rats exhibited an orienting response to the whitenoise CS, a response that competes with freezing behaviour. It is therefore likely that our measure of freezing during the 2-s white noise underestimated the degree of conditional fear to the white noise.

Surprisingly, context freezing in the paired rats was also greater than that in the unpaired rats. This result was unexpected insofar as signalling USs with discrete CSs typically reduces context freezing compared to the condition in which the cues are explicitly unpaired. It is unclear why we observed this pattern of results, although it is worth noting that, compared to the paired rats, unpaired rats showed relatively greater levels of freezing to the context vs. the white-noise CS.

It is important to note that 75 conditioning trials produced asymptotic conditional freezing to the white-noise CS in the paired rats. Indeed, we have found in pilot experiments that 25 conditioning trials are sufficient to produce asymptotic freezing. Therefore, our experiments reveal that significant conditioning-related increases in 
CS-elicited unit activity are evident in LAd neurons after levels of training that far exceed those necessary to produce asymptotic behavioural performance.

\section{Discussion}

The present results confirm earlier reports of conditioning-related increases in auditory CS-elicited spike firing in LAd (Quirk et al., 1995, 1997), and extend these reports by demonstrating that these changes also occur in extensively overtrained rats. Increases in CSelicited spike firing in LAd neurons were associative in nature, insofar as 75 unpaired noise-shock trials did not significantly elevate CS-elicited spike firing. Moreover, neuronal plasticity in LAd was correlated with the development and expression of conditional freezing behaviour. Consistent with earlier reports (Quirk et al., 1995, 1997), associative increases in LAd firing occurred at short latencies $(<20 \mathrm{~ms})$ from $\mathrm{CS}$ onset, which implicates the direct thalamo-amygdala pathway in this form of learning-induced plasticity. Interestingly, we have also found that extensive overtraining further sharpens spike firing within the short-latency bins, suggesting that plasticity in the thalamo-amygdala, rather than cortico-amygdala, pathway is responsible for associative increases in LAd firing in both minimally and extensively trained rats.

Although we have found increases in CS-elicited amygdaloid unit firing that persist despite extensive overtraining, earlier reports indicate that associative increases in BLA unit firing dissipate under similar conditions. For example, we previously reported that learningrelated increases in multiple-unit activity in the BLA dissipate over the course of extensive overtraining in an avoidance conditioning paradigm in rabbits (Maren etal., 1991). In the light of the present results, we suggest that decreases in associative firing in the avoidance task relate to a shift during training in the associative processes that mediate avoidance learning (see Maren, 1998 for a discussion), that is, early in avoidance learning animals learn a Pavlovian association between the auditory CS and footshock. It is during this phase of training that avoidance conditioning is amygdaladependent (e.g. Brady et al., 1954; Poremba \& Gabriel, 1997, 1999) and conditioning-related increases in CS-elicited firing in the amygdala are the largest (Maren et al., 1991). As avoidance learning progresses, the Pavlovian association gives way to an instrumental association (e.g. Mineka \& Gino, 1980) that is independent of the amygdala. Thus, associative increases in CS-elicited firing dissipate after overtraining in the avoidance task (Maren etal., 1991), and amygdala lesions (e.g. Brady et al., 1954) or inactivation (Poremba \& Gabriel, 1999) made after overtraining do not affect expression of the overtrained avoidance response.

Other investigators have reported transient decreases in amygdala activity during Pavlovian fear conditioning in both rats and humans (Quirk etal., 1997; Buchel etal., 1998; LaBar etal., 1998). These decreases are only evident during the conditioning session itself; that is, robust associative increases in CS-elicited activity re-emerge during extinction testing after the conditioning session is completed. The mechanisms underlying these within-session decreases in amygdaloid neuronal activity during Pavlovian fear conditioning are not presently known, but they do not appear to reflect the disengagement of the amygdala that has been observed in avoidance learning studies (e.g. Maren etal., 1991). Indeed, the results of the present study indicate that LAd neurons continue to exhibit robust fear conditioning-related increases in spike firing even after extensive overtraining. One possibility is that amygdala neurons are involved in detecting changes in the CS-US contingency, which occur early in both conditioning and extinction (Quirk etal., 1997).
Our analysis of the latency of peak CS-elicited firing reveals that overtraining focuses plasticity in the direct thalamo-amygdala pathway. This was evident by the fact that peak associative firing occurred in LAd within $20 \mathrm{~ms}$ of CS onset after overtraining. Importantly, these data indicate that associative plasticity in the amygdala is not merely relayed from the auditory cortex, as might be predicted by models that posit memory storage in the neocortex (e.g. Weinberger, 1993; McGaugh, 2000). However, an outstanding question is whether the associative increases in CS-elicited spike firing observed in LAd are mediated by plasticity at thalamoamygdala synapses, or whether they reflect plasticity in brain structures afferent to the amygdala. It is well documented that fear conditioning induces plasticity in the medial geniculate nucleus (Edeline \& Weinberger, 1992; Supple \& Kapp, 1989; McEchron etal., 1995), which is the primary thalamic afferent for auditory projections to the amygdala (LeDoux et al., 1990; Doron \& LeDoux, 1999). Therefore, it is plausible that associative plasticity is established in the thalamus and then relayed to the LA (Cahill et al., 1999). Nonetheless, there is evidence to suggest that thalamoamygdala synapses exhibit plasticity during fear conditioning (McKernan \& Shinnick-Gallagher, 1997; Rogan et al., 1997), which might be a mechanism to support conditioning-related plasticity in LA neurons. Moreover, manipulations that disrupt amygdaloid longterm potentiation (LTP), a putative synaptic mechanism for memory storage in the amygdala (Maren, 1999b), attenuate the acquisition of fear conditioning (Miserendino et al., 1990; Maren et al., 1996b; Lee \& Kim, 1998; Goosens etal., in press). Clearly, further work is required to delineate the essential locus (or loci) of plasticity supporting associative changes in amygdala spike firing after fear conditioning.

In either case, the existence of short-latency associative neuronal discharges in LAd after extensive overtraining is consistent with the view that the amygdala has an enduring role in mediating a memory for Pavlovian fear conditioning (Davis, 1997; Fanselow \& LeDoux, 1999; Maren \& Fanselow, 1996; LeDoux, 2000). In contrast to this view, it has been suggested (e.g. McGaugh, 2000) that the role of the amygdala in aversive learning is temporary and is limited by either the passage of time (Liang et al., 1982) or extensive training (Parent et al., 1992, 1994; Mesches et al., 1996). These conclusions have been drawn from studies of inhibitory avoidance conditioning, a form of aversive learning that involves both Pavlovian and instrumental associations. However, there is abundant evidence indicating that the role of the BLA in Pavlovian fear conditioning is not limited by either time (Lee etal., 1996; Maren etal., 1996a) or degree of training (Maren, 1998; Maren, 1999a). Therefore, we suggest that the nature of the aversive conditioning task is an important determinant of amygdaloid involvement in aversive learning. Specifically, we propose that the amygdala has an enduring role in encoding and storing Pavlovian associations within the amygdala, but has a temporary role in encoding and modulating the storage of instrumental associations, the memories for which are presumed to reside outside of the amygdala. A strong prediction of this model is that overtraining in an instrumental avoidance task, for example, will mitigate the effects of BLA lesions on avoidance responding, but not on Pavlovian fear responses. Thus, we predict that the BLA is only essential for aversive conditioning when Pavlovian associations mediate the learned response (in this case, conditional freezing). We are currently performing experiments to test this prediction.

The present neurophysiological results converge with the results from a recent report we published indicating that the BLA is essential for both the acquisition and expression of auditory fear conditioning even after extensive overtraining (Maren, 1998; Maren, 1999a). It 
should be noted, however, that a recent report found that pretraining BLA lesions did not affect the acquisition of auditory fear conditioning when conditioned suppression (rather than freezing) was used to assess fear (Killcross et al., 1997). In this study, rats learned both instrumental and Pavlovian associations, and animals with BLA lesions were selectively impaired at learning the instrumental association. These data are perplexing in view of the large literature implicating the BLA in the acquisition of fear conditioning to auditory CSs (e.g. Selden etal., 1991; Campeau \& Davis, 1995; Maren etal., 1996a; Maren, 1999a; Amorapanth et al. 2000). However, it is possible that the response contingency for CS presentation in these experiments (CS presentation was initiated by the rat, not the experimenter) altered the involvement of the BLA in fear conditioning (see also Nader \& LeDoux, 1997). Indeed, in a more standard suppression task (one in which CS presentation was initiated by the experimenter, not the rat) BLA lesions have been found to impair conditioned suppression to an auditory CS even after extensive overtraining (Sun etal., 1999). Thus, it appears that the BLA is in fact essential for auditory fear conditioning even after extensive overtraining in standard Pavlovian fear conditioning paradigms.

In conclusion, we have demonstrated that auditory fear conditioning induces robust increases in short-latency CS-elicited spike firing in LAd neurons even after extensive overtraining. The persistence of neurophysiological plasticity in LAd neurons after extensive overtraining is consistent with the proposal that BLA neurons are an essential locus of plasticity in Pavlovian fear conditioning (Maren \& Fanselow, 1996; Fanselow \& LeDoux, 1999; LeDoux, 2000). The mechanisms underlying conditioning-related increases in CS-elicited firing in LAd neurons are presently unknown, but may involve cellular processes thought to mediate fear conditioning such as amygdaloid long-term potentiation (Maren, 1999b).

\section{Acknowledgements}

This work was supported by grants from the National Institute of Mental Health (R29MH57865) and the University of Michigan.

\section{Abbreviations}

AST, amygdalo-striatal transition zone; BLA, basolateral complex of the amygdala; CS, conditional stimulus; LA, lateral amygdaloid nucleus; LAd, lateral amygdaloid nucleus, dorsal division; LTP, long-term potentiation; PSTH, poststimulus time histogram; US, unconditional stimulus.

\section{References}

Amorapanth, P., LeDoux, J.E. \& Nader, K. (2000) Different lateral amygdala outputs mediate reactions and actions elicited by a fear-arousing stimulus. Nature Neurosci., 3, 74-79.

Brady, J.V., Schreiner, L., Geller, I. \& Kling, A. (1954) Subcortical mechanisms in emotional behavior. The effect of rhinencephalic injury upon the acquisition and retention of a conditioned avoidance response in cats. J. Comp. Physiol. Psychol., 47, 179-186.

Buchel, C., Morris, J., Dolan, R.J. \& Friston, K.J. (1998) Brain systems mediating aversive conditioning. an event-related fMRI study. Neuron, 20, 947-957.

Cahill, L., Weinberger, N.M., Roozendaal, B. \& McGaugh, J.L. (1999) Is the amygdala a locus for 'conditioned fear'? Some questions and caveats. Neuron, 23, 227-228.

Cahill, L. \& McGaugh, J.L. (1998) Mechanisms of emotional arousal and lasting declarative memory. Trends Neurosci., 21, 294-299.

Campeau, S. \& Davis, M. (1995) Involvement of the central nucleus and basolateral complex of the amygdala in fear conditioning measured with fear-potentiated startle in rats trained concurrently with auditory and visual conditioned stimuli. J. Neurosci., 15, 2301-2311.
Davis, M. (1997) Neurobiology of fear responses. The role of the amygdala. $J$. Neuropsychiat. Clin. Neurosci., 9, 382-402.

Doron, N.N. \& LeDoux, J.E. (1999) Organization of projections to the lateral amygdala from auditory and visual areas of the thalamus in the rat. J. Comp. Neurol., 412, 383-409.

Edeline, J.-M. \& Weinberger, N.M. (1992) Associative retuning in the thalamic source of input to the amygdala and auditory cortex. Receptive field plasticity in the medial division of the medial geniculate body. Behav. Neurosci., 106, 81-105.

Fanselow, M.S. \& LeDoux, J.E. (1999) Why we think plasticity underlying Pavlovian fear conditioning occurs in the basolateral amygdala. Neuron, 23, 229-232.

Goosens, K.A., Holt, W. \& Maren, S. (2000) A role for amygdaloid PKA and $\mathrm{PKC}$ in the acquisition of conditional fear memories in rats. Behav. Brain Res., 114, 145-152.

Killcross, S., Robbins, T.W. \& Everitt, B.J. (1997) Different types of fearconditioned behaviour mediated by separate nuclei within amygdala. Nature (Lond.), 388, 377-380.

LaBar, K.S., Gatenby, J.C., Gore, J.C., LeDoux, J.E. \& Phelps, E.A. (1998) Human amygdala activation during conditioned fear acquisition and extinction. A mixed-trial fMRI study. Neuron, 20, 937-945.

LeDoux, J.E. (2000) Emotion circuits in the brain. Annu. Rev. Neurosci., 23, $155-184$.

LeDoux, J.E., Farb, C. \& Ruggiero, D.A. (1990) Topographic organization of neurons in the acoustic thalamus that project to the amygdala. J. Neurosci., 10, 1043-1054.

Lee, H. \& Kim, J.J. (1998) Amygdalar NMDA receptors are critical for new fear learning in previously fear-conditioned rats. J. Neurosci., 18, 84448454.

Lee, Y., Walker, D. \& Davis, M. (1996) Lack of a temporal gradient of retrograde amnesia following NMDA-induced lesions of the basolateral amygdala assessed with the fear-potentiated startle paradigm. Behav. Neurosci., 110, 836-839.

Liang, K.C., McGaugh, J.L., Martinez, J.L. Jr, Jensen, R.A., Vasquez, B.J. \& Messing, R.B. (1982) Post-training amygdaloid lesions impair retention of an inhibitory avoidance response. Behav. Brain Res., 4, 237-249.

Maren, S. (1998) Overtraining does not mitigate contextual fear conditioning deficits produced by neurotoxic lesions of the basolateral amygdala. $J$. Neurosci., 18, 3088-3097.

Maren, S. (1999a) Neurotoxic basolateral amygdala lesions impair learning and memory but not the performance of conditional fear in rats. $J$. Neurosci., 19, 8696-8703.

Maren, S. (1999b) Long-term potentiation in the amygdala: a mechanism for emotional learning and memory. Trends Neurosci., 22, 561-567.

Maren, S., Aharonov, G. \& Fanselow, M.S. (1996a) Retrograde abolition of conditional fear after excitotoxic lesions in the basolateral amygdala of rats. Absence of a temporal gradient. Behav. Neurosci., 110, 718-726.

Maren, S., Aharonov, G., Stote, D.L. \& Fanselow, M.S. (1996b) N-methyl-Daspartate receptors in the basolateral amygdala are required for both acquisition and expression of conditional fear in rats. Behav. Neurosci., 110, $1365-1374$

Maren, S. \& Fanselow, M.S. (1996) The amygdala and fear conditioning: has the nut been cracked? Neuron, 16, 237-240.

Maren, S., Poremba, A. \& Gabriel, M. (1991) Basolateral amygdaloid multiunit neuronal correlates of discriminative avoidance learning in rabbits. Brain Res., 549, 311-316.

McEchron, M.D., McCabe, P.M., Green, E.J., Llabre, M.M. \& Schneiderman, N. (1995) Simultaneous single unit recording in the medial nucleus of the medial geniculate nucleus and amygdaloid central nucleus throughout habituation, acquisition, and extinction of the rabbit's classically conditioned heart rate. Brain Res., 682, 157-166.

McKernan, M.G. \& Shinnick-Gallagher, P. (1997) Fear conditioning induces a lasting potentiation of synaptic currents in vitro. Nature (Lond), 390, 607611.

McGaugh, J.L. (2000) Memory - a century of consolidation. Science, 287, 248-251.

Mesches, M.H., Bianchin, M. \& McGaugh, J.L. (1996) The effects of intraamygdala infusion of the AMPA receptor antagonist CNQX on retention performance following aversive training. Neurobiol. Learn. Mem., 66, 324340 .

Mineka, S. \& Gino, A. (1980) Dissociation between conditioned emotional response and extended avoidance performance. Learn. Motiv., 11, 476-502.

Miserendino, M.J., Sananes, C.B., Melia, K.R. \& Davis, M. (1990) Blocking of acquisition but not expression of conditioned fear-potentiated startle by NMDA antagonists in the amygdala. Nature (Lond.), 345, 716-718. 
McNaughton, B.L., O'Keefe, J. \& Barnes, C.A. (1983) The stereotrode. a new technique for simultaneous isolation of several single units in the central nervous system from multiple unit records. J. Neurosci. Meth., 8, 391-397.

Nader, K. \& LeDoux, J.E. (1997). Is it time to invoke multiple learning systems in the amygdala? Trends Cogn. Sci., 1, 241-244.

Parent, M.B., Tomaz, C. \& McGaugh, J.L. (1992) Increased training in an aversively motivated task attenuates the memory-impairing effects of posttraining N-methyl-D-aspartate-induced amygdala lesions. Behav. Neurosci., 106, 789-797.

Parent, M.B., West, M. \& McGaugh, J.L. (1994) Memory of rats with amygdala lesions induced 30 days after footshock-motivated escape training reflects degree of original training. Behav. Neurosci., 108, 1080-1087.

Poremba, A. \& Gabriel, M. (1997) Amygdalar lesions block discriminative avoidance learning and cingulothalamic training-induced neuronal plasticity in rabbits. J. Neurosci., 17, 5237-5244.

Poremba, A. \& Gabriel, M. (1999) Amygdala neurons mediate acquisition but not maintenance of instrumental avoidance behavior in rabbits. J. Neurosci. 19, 9635-9641.

Quirk, G.J., Repa, C. \& LeDoux, J.E. (1995) Fear conditioning enhances shortlatency auditory responses of lateral amygdala neurons. Parallel recordings in the freely behaving rat. Neuron, 15, 1029-1039.
Quirk, G.J., Armony, J.L. \& LeDoux, J.E. (1997) Fear conditioning enhances different temporal components of tone-evoked spike trains in auditory cortex and lateral amygdala. Neuron, 19, 613-624.

Rogan, M.T., Staubli, U.V. \& LeDoux, J.E. (1997) Fear conditioning induces associative long-term potentiation in the amygdala. Nature (Lond.), 390, 604-607.

Selden, N.R., Everitt, B.J., Robbins, T.W. \& Jarrard, L.E. (1991) Complementary roles for the amygdala and hippocampus in aversive conditioning to explicit and contextual cues. Neuroscience, 42, 335-350.

Sun, P., Nader, K. \& LeDoux, J.E. (1999) The basolateral amygdala is required for auditory conditioned suppression even in animals that are overtrained. Soc., Neurosci. Abstr., 25, 1618.

Supple, W.F. \& Kapp, B.S. (1989) Response characteristics of neurons in the medial component of the medial geniculate nucleus during Pavlovian differential fear conditioning in rabbits. Behav. Neurosci., 103, 1276-1286.

Weinberger, N.M. (1993) Learning-induced changes of auditory receptive fields. Curr. Opin. Neurobiol., 3, 570-577.

Wilson, M.A. \& McNaughton, B.L. (1993) Dynamics of the hippocampal ensemble code for space. Science, 261, 1055-1058. 